Nouvelles perspectives en sciences sociales

\title{
Analyse statistique linéaire et interprétation systémique
}

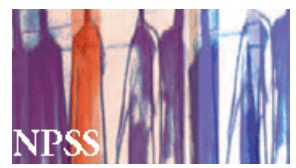

\section{Simon Laflamme}

Volume 4, numéro 1, septembre 2008

URI : https://id.erudit.org/iderudit/019642ar

DOI : https://doi.org/10.7202/019642ar

Aller au sommaire du numéro

\section{Éditeur(s)}

Prise de parole

\section{ISSN}

1712-8307 (imprimé)

1918-7475 (numérique)

Découvrir la revue

\section{Citer cet article}

Laflamme, S. (2008). Analyse statistique linéaire et interprétation systémique. Nouvelles perspectives en sciences sociales, 4(1), 141-159.

https://doi.org/10.7202/019642ar

\section{Résumé de l'article}

La plupart des analyses statistiques sont construites selon une logique linéaire : analyses de variance, analyses de régression. Pourtant, bon nombre de ces analyses dépeignent des phénomènes dont l'interprétation théorique est impossible dans une logique linéaire; ces phénomènes appellent plutôt une théorisation de type dialectique. Plus encore : les techniques statistiques linéaires permettent communément des modélisations multivariées. Or, les associations entre variables qu'elles décrivent, même si elles sont statistiquement soumises à une logique linéaire, permettent aisément des interprétations systémiques. Ainsi, la linéarité de l'analyse statistique peut servir des théorisations relationnelles ou systémiques. C'est ce que voudrait illustrer cet article. Pour ce faire, il présentera les résultats de deux analyses empiriques effectuées avec des outils statistiques sur des phénomènes sociaux (rapport des jeunes à la communauté, usages des médias) et il montrera comment les objets qui sont dépeints ne peuvent être théorisés adéquatement sans qu'on recoure à des notions de récursivité, de dialectique et de système. 


\title{
Analyse statistique linéaire et interprétation systémique
}

\author{
SimON LAFLAMmE \\ Université Laurentienne
}

$\mathrm{C}_{\text {qui sciences sociales, l'analyse systémique, notamment celle }}$ gié l'analyse qualitative, tenant l'analyse statistique comme réductrice des phénomènes sociaux en tant surtout qu'elle serait trop linéaire pour faire état des dynamiques qui caractérisent la socialité. L'article qui est ici livré se veut une critique de cet antiquantitativisme. Dans un premier temps, il met en lumière les principes qui animent ce rejet de la statistique et il en montre la fragilité. Dans un second temps, il propose deux exemples d'analyses statistiques sur lesquelles se sont édifiées des interprétations systémiques.

\section{Notions de systémique}

\subsection{Systémique et empirie}

La systémique consiste souvent à organiser de l'information. Elle est principalement à l'œuvre quand il s'agit d'établir des liens entre les concepts. Dans les sciences sociales, elle est nettement plus théorique qu'empirique.

La systémique est aussi construction du monde, bien sûr, comme l'est toute approche analytique. Mais elle construit 
souvent à distance, son rapport avec l'empirie étant ténu. Ce n'est pas sans raison si, historiquement, en sciences sociales tout au moins, elle est plus à son aise dans les tâches d'interprétation que dans celles d'observation.

La systémique sert parfois des entreprises de modélisation, c'est-à-dire, au sens d'Yvon Gauthier ${ }^{1}$, qu'elle est utile à la fabrication de ponts entre la théorie et l'empirie. Elle est alors moins strictement interprétative.

\subsection{Systémique et complexité}

En sciences sociales, systémique rime souvent avec complexité. La systémique apparaît alors comme une tentative de faire état du caractère irréductible du monde; elle se fait critique des interprétations qui apparaissent trop simplificatrices.

Consciente des dangers de la simplification, la systémique complexe se révèle souvent comme un projet de totalisation. Les reproches qu'elle adresse aux travaux de recherche qui sont réalisés ailleurs se manifestent souvent comme des dénonciations de ce qui a été omis, comme des constats d'incomplétude ${ }^{2}$.

Cette critique rend la systémique suspicieuse à l'égard de l'observation des objets sociaux et, plus généralement, humains. Consciente de la complexité du monde, il lui est difficile d'oser une observation, laquelle serait forcément simplification.

\subsection{Systémique, complexité, subjectivité}

Dans les sciences sociales, les chercheurs qui prônent la complexité s'intéressent, bien sûr, au lien entre les objets analysés; mais ils tendent surtout à se pencher sur la subjectivité, le social et l'humain se révélant par-dessus tout dans la subjectivité. Ainsi, omettre la subjectivité, c'est ne pas comprendre le social et l'humain. Ainsi, encore, se concentrer sur la subjectivité, c'est faire de la complexité. En ce sens, les sciences sociales deviennent

Yvon Gauthier, Entre science et culture. Introduction à la philosophie des sciences, Montréal, Presses de l'Université de Montréal, 2005.

2 Edgar Morin et Jean Louis Le Moigne, L'intelligence de la complexité, Paris, L'Harmattan, coll. "Cognition et formation », 1999. 
d'autant plus respectueuses de l'empirie qu'elles n'omettent pas la subjectivité.

La systémique complexe n'est pas la seule à véhiculer cette idéologie analytique; toutes les sociologies centrées sur le sujet font de même : interactionnisme symbolique, ethnométhodologie, analyse conversationnelle, individualisme méthodologique. Ce qui distingue la systémique de ces approches, ce n'est pas l'impératif de subjectivité, c'est l'obligation de réunir l'information dans un système.

\subsection{Distorsion}

Ces critiques ont su mettre au jour de graves lacunes dans plusieurs travaux sociologiques. On leur doit, par exemple, d'avoir mis en évidence des abus d'analyses statistiques ou de théorisations de comportements humains.

On leur doit, par contre, des épistémologies naïves qui les ont empêchées de relever dans les travaux inspirés de la systémique complexe ce qu'elles notaient chez les autres. En dénonçant, par exemple, des quantifications du monde, en reprochant à ces quantifications de provoquer des " distorsions ", la systémique complexe ne voyait pas qu'elle était elle-même construction du monde, et donc qu'elle procédait, elle-même, à des " distorsions ". La théorie de l'acteur rationnel dont elle se nourrit communément - comme presque toutes les sociologies centrées sur l'individu - en est un bel exemple. Les concepts de " dialogie " ou de " récursivité " sont construction. Nous signalons au passage que cette épistémologie laisse entendre qu'il y a une manière d'aborder le monde sans créer de "distorsion " et qu' elle renvoie ainsi indirectement à un objectivisme - qu'elle dénonce par ailleurs.

\subsection{Deux propositions}

Dans ses tentatives d'opérationnaliser, la systémique complexe a abordé le monde humain en se tournant vers la subjectivité et, pour ce faire, elle a privilégié des modélisations qualitatives. Mais sont sous-entendues dans cette attitude deux propositions critiquables : 
i. les sciences sociales sont d'autant plus complexes qu'elles examinent la subjectivité;

ii. les sciences sociales sont d'autant plus complexes qu'elles modélisent leur objet de façon qualitative.

\subsection{Pour la systémique complexe}

Il nous apparaît pourtant :

i. que la subjectivité est certes complexe, mais qu'on peut très bien ne pas la comprendre dans une logique complexe;

ii. qu'on peut faire de la systémique, même complexe, sans avoir pour centre la subjectivité;

iii. que la subjectivité peut être comprise aussi bien de façon qualitative que de façon quantitative;

iv. que toute analyse qualitative n'est pas propre à servir une analyse complexe;

v. que l'analyse statistique peut servir la systémique complexe.

En fait, c'est plus la manière d'aborder le monde et de l'analyser qui conduit vers la complexité que l'objet analysé. La systémique n'est pas une science de la totalité, sinon elle n'est pas une science. Il n'y a de science que du circonscrit.

\subsection{Systémique et statistique}

La systémique se veut par-dessus tout une science du lien. Ses maîtres-mots sont " récursivité ", " interrelation ", " dialectique ", " dialogie ", " organisation »... Pour cette raison, elle se méfie des logiques linéaires, des modèles causaux, des analyses unidirectionnelles.

La statistique peut éveiller cette méfiance comme elle peut aussi prêter le flanc à la critique qui l'accompagne. Mais elle est aussi victime d'une méfiance et d'une critique qui sont injustifiées.

\subsection{Statistique et linéarité}

La statistique travaille souvent dans une logique linéaire. Mais la linéarité, chez elle, n'est pas du registre de l'orientation du lien. 
Elle est de celui de la ligne. La statistique linéaire n'est pas celle qui pose que les relations entre les variables sont unidirectionnelles. C'est celle qui transforme en lignes les relations entre variables pour en comprendre l'association. Ce n'est pas la même chose. La notion de causalité n'appartient pas à la statistique, elle appartient aux théories qui recourent à elle. L'essence de la statistique, c'est la covariation. Plus est forte la covariation entre variables, plus sont liées ces variables. En ce sens, la statistique est très proche des principes de la systémique pour laquelle les modèles causaux sont rares, pour laquelle les corrélations sont habituelles. La covariation est corrélation. Pour qu'il y ait corrélation (" interrelation ", "dialectique ", " récursivité »), il faut qu'il y ait covariation. Établir dans quelle mesure deux variables sont associées de façon linéaire, forment ou non une ligne, ce n'est pas préjuger de la causalité de la relation, ce n'est pas affirmer que ces variables sont associées de telle manière qu'elles appartiennent à l'unidirectionnalité.

\subsection{Statistique et complexité}

En fonction de ce qui la caractérise, la statistique est à même d'aider les théorisations systémiques. En aval comme en amont.

Elle peut aussi aider non seulement parce qu'elle est de l'ordre de la covariation, mais en plus parce quelle fournit bon nombre d'informations dont l'interprétation la dépasse, toujours parce qu'elles appellent la théorisation, parce que les informations sont tellement complexes qu'elles demandent une interprétation systémique, voire systémique complexe.

\subsection{Parenté du qualitatif et du quantitatif}

Les variables, en statistique, sont souvent les indicateurs de catégories plus abstraites. Elles peuvent donner lieu à des conceptualisations ou à des catégorisations. Il en va de même en analyse qualitative où, par exemple, dans la manipulation de données textuelles, les énoncés donnent lieu à des catégories et les catégories appellent à être reliées entre elles.

Le mouvement analytique, ici, n'est pas à sens unique. On 
peut aller de la théorie aux observations et inversement. Il peut y avoir des va-et-vient (voir la figure 1).

Analytiquement et scientifiquement, modélisation qualitative et modélisation quantitative sont comparables - quoi qu'en disent les idéologies qualitativiste ou quantitativiste -, pourvu que toutes deux se soumettent aux impératifs de la rigueur.

\section{Figure 1}

\section{Opérations relevant aussi bien de l'analyse qualitative que de l'analyse quantitative}

$\Uparrow$

Construction de relations entre catégories (théorisation) Création de catégories (modélisation)

Observation empirique (variables, indicateurs, énoncés...)

\section{Deux illustrations}

Deux études peuvent illustrer cette compatibilité entre l'analyse statistique et l'interprétation systémique : dans la première sont principalement comparées des moyennes et, dans la seconde, sont corrélées des variables de façon régressive. Il s'agit, en fait, de deux des opérations les plus communes en statistique, une troisième étant la comparaison de fréquences absolues ou relatives en fonction de modalités.

\subsection{Autour d'une analyse de variance}

Dans cette première analyse, il s'agit de vérifier si la profession, définie d'après le secteur et le niveau, a une incidence sur le rapport aux médias, l'identité et l'engagement social ${ }^{3}$.

3 Ces données sont tirées d'une enquête menée dans le Nord-Ouest de l'Ontario par Chris Southcott, Nicole Corbett et Simon Laflamme, financée par le Conseil de recherche en sciences humaines du Canada. Les analyses seront publiées dans «Le rapport aux médias et la profession exercée. Le cas des 
Un premier tableau examine la fréquence des activités qui sont propres aux médias plutôt traditionnels comme la lecture des journaux imprimés, des magazines imprimés ou des ouvrages littéraires; l'écoute de la radio; l'exposition à la télévision. Il compare cette fréquence selon le domaine de la profession et selon son niveau. La plupart des valeurs de " $\mathrm{p}$ " sont supérieures à 0,05 , ce qui révèle que, la plupart du temps, il n'y a pas d'inégalités de moyennes; cette inégalité peut toutefois survenir, comme c'est le cas pour la lecture d'ouvrages littéraires, quand elle est comparée selon le secteur de la profession, ou de la lecture de journaux imprimés quand intervent le niveau professionnel. On aura à l'esprit que les valeurs se situent entre « 1 » et « 6 ", " 1 » signifiant « jamais » et " 6 », " très souvent ». Si l'on observe les moyennes, on constate, en effet, qu'elles sont normalement semblables d'une population à l'autre et qu'elles varient d'une activité à l'autré

francophones du Nord-Ouest de l'Ontario », de Simon Laflamme et Chris Southcoutt, à paraître.

4 Pour ne pas alourdir l'exposé, les effets d'interaction, tels que signalés par l'analyse de variance, ne sont pas pris en considération. 


\begin{tabular}{|c|c|c|c|c|c|c|c|c|c|c|c|c|c|c|}
\hline & Fré & $\begin{array}{r}\text { quer } \\
\text { sel }\end{array}$ & $\begin{array}{l}\text { ce } n \\
\text { on } 1\end{array}$ & $\begin{array}{l}\text { oyer } \\
\text { sect } \\
(1=\end{array}$ & $\begin{array}{l}\text { ne d } \\
\text { eur et } \\
\text { jama }\end{array}$ & $\begin{array}{l}\text { di } \\
\text { le } \\
\text { s; } 6\end{array}$ & $\begin{array}{l}\text { au } 1 \\
\text { erses } \\
\text { lvea } \\
=\text { très }\end{array}$ & $\begin{array}{l}\text { activi } \\
\text { de la } \\
\text { souven }\end{array}$ & c) & édi & 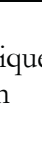 & & & \\
\hline Activité & & & & Secte & ur de & $\mathrm{apr}$ & Eessic & & & & eau & e la $p$ & ofes & \\
\hline & & 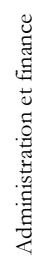 & 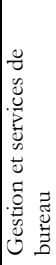 & 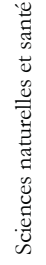 & 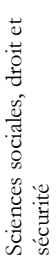 & 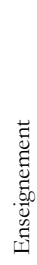 & $\stackrel{\Xi}{\Xi}$ & 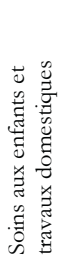 & 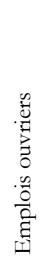 & 节 & 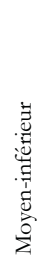 & है & 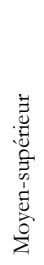 & 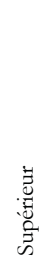 \\
\hline Je lis des & \begin{tabular}{|l|}
$\bar{X}$ \\
\end{tabular} & 4,47 & \begin{tabular}{|l|}
4,30 \\
\end{tabular} & 4,50 & \begin{tabular}{|l|}
4,17 \\
\end{tabular} & 4,64 & 3,68 & 4,06 & 3,87 & 4,09 & 4,31 & 4,58 & 4,22 & $\overline{4,50}$ \\
\hline $\begin{array}{l}\text { journaux } \\
\text { imprimés }\end{array}$ & test & & & & $2399=1$ & $22 ; \mathrm{p}$ & 0,29 & & & & $\mathrm{~F}_{(4 ; 239)}$ & 2,57 & $<0,05$ & \\
\hline Je lis des revues & $\bar{X}$ & 4,63 & \begin{tabular}{|l|}
4,35 \\
\end{tabular} & 4,58 & \begin{tabular}{|l|}
4,43 \\
\end{tabular} & 4,86 & 3,68 & 4,29 & 3,81 & 4,14 & 4,40 & 4,55 & 4,67 & 4,17 \\
\hline $\begin{array}{l}\text { magazines } \\
\text { imprimés }\end{array}$ & test & & & & 40) $=1$ & 40. & $=0,19$ & & & & 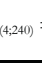 & 170 & $=0$, & \\
\hline Je lis des & \begin{tabular}{|l|}
$\bar{X}$ \\
\end{tabular} & 4,37 & \begin{tabular}{|l|}
4,14 \\
\end{tabular} & 4,04 & \begin{tabular}{|l|}
3,68 \\
\end{tabular} & 4,89 & 2,96 & 4,65 & 2,66 & 3,50 & 3,62 & 4,12 & 4,71 & 4,17 \\
\hline $\begin{array}{l}\text { ouvrages } \\
\text { littéraires }\end{array}$ & test & & & $\mathrm{F}_{(7 ;}$ & $36)=3$ & 05 ; & $<0,0$ & & & & ;236) $=$ & 0,50 & $=0$ & \\
\hline J'écoute de la & 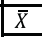 & 4,32 & 5,23 & 4,22 & \begin{tabular}{|l|}
4,32 \\
\end{tabular} & 4,91 & 4,46 & 4,76 & 3,87 & 4,47 & 4,44 & 4,12 & 4,82 & 4,50 \\
\hline $\begin{array}{l}\text { musique à } \\
\text { domicile }\end{array}$ & test & & & & ;239) $=1$ & $03 ; 1$ & 0,41 & & & & $(4 ; 239)=$ & 0,41 ; & $=0$, & \\
\hline J'écoute la & $\bar{X}$ & 5,00 & \begin{tabular}{|l|}
5,27 \\
\end{tabular} & 4,63 & \begin{tabular}{|l|l|}
4,77 \\
\end{tabular} & 4,50 & 4,57 & 4,71 & 4,50 & 4,85 & 4,64 & 4,85 & 4,51 & 4,50 \\
\hline & test & & & & $237)=0$ & $64 ; 1$ & $=0,73$ & & & & $\overline{(4 ; 237)}=$ & 0,73 ; & $=0$ & \\
\hline Je regarde la & 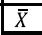 & 5,06 & 4,68 & 4,89 & \begin{tabular}{|l|l|}
4,83 \\
\end{tabular} & 4,60 & 5,04 & 5,12 & 4,77 & 5,14 & 4,70 & 4,58 & 4,68 & 4,58 \\
\hline & test & & & & :237) $=0$ & $32 ; 1$ & $=0,95$ & & & & $(4 ; 237)=$ & 0,52 ; & $=0$, & \\
\hline Je regarde des & 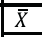 & 4,16 & \begin{tabular}{|l|}
4,45 \\
\end{tabular} & 4,24 & \begin{tabular}{|l|}
3,93 \\
\end{tabular} & 4,16 & 4,21 & 5,12 & 4,18 & 4,42 & 4,08 & 4,13 & 4,24 & 3,42 \\
\hline $\begin{array}{l}\text { enregistrements } \\
\text { vidéo }\end{array}$ & test & & & & $232)=$ & 92 & $=0,49$ & & & & $(4,232$ & 0,45 ; & $=0$ & \\
\hline J'utilise un & $\bar{X}$ & 2,94 & \begin{tabular}{|l|}
2,64 \\
\end{tabular} & 2,89 & \begin{tabular}{|l|}
2,98 \\
\end{tabular} & 2,31 & 2,89 & 3,29 & 2,43 & 2,93 & 2,69 & 3,16 & 2,49 & 1,75 \\
\hline $\begin{array}{l}\text { ordinateur pour } \\
\text { jouer }\end{array}$ & test & & & & $235)=0$ & $5 ; 1$ & $=0,47$ & & & & $(4,235)=$ & 1,27 & $=0$ & \\
\hline
\end{tabular}

Un deuxième tableau reprend la comparaison selon le domaine et le niveau professionnel, mais, cette fois, pour divers usages d'Internet (le tableau ne rapporte que 8 des 33 usages qui ont été soumis aux analyses de variance). Il permet de constater que parfois les moyennes sont égales, parfois non. Il ne révèle pas de manière univoque que plus le statut de sa profession est élevé, plus l'individu tend à recourir à Internet. On découvre à nouveau dans ce tableau que les moyennes varient considérablement d'un usage à l'autre, plus qu'elles ne le font entre les secteurs ou les niveaux professionnels. 


\begin{tabular}{|c|c|c|c|c|c|c|c|c|c|c|c|c|c|c|}
\hline & & & aen & e 1 & $\begin{array}{l}\mathrm{T} \\
\text { yenne } \\
\text { ur et } 1 \\
\text { jamais }\end{array}$ & $\begin{array}{l}\text { ablea } \\
\text { de di } \\
\text { le niv } \\
; 6=\end{array}$ & $\begin{array}{l}2 \\
\text { vers } \\
\text { cau d } \\
\text { rès so }\end{array}$ & $\begin{array}{l}\text { sages } \\
\text { la } \mathrm{pr} \\
\text { vent) }\end{array}$ & T, & rnet & & & & \\
\hline Usage d'Internet & & & & & ur de la & profe & ssion & & & & iveau & e lap & ofessi & \\
\hline & & 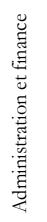 & 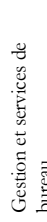 & 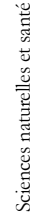 & 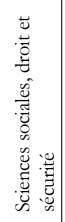 & 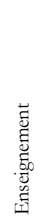 & 苛 & 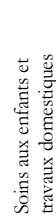 & 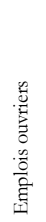 & 莺 & 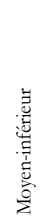 & 芯 & 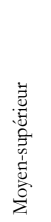 & 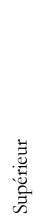 \\
\hline J'utilise Internet & $\bar{X}$ & 4,17 & 3,61 & 3,7 & 3,27 & 4,14 & 2,55 & 4,82 & 3,38 & 3,28 & 3,56 & 4,35 & 3,85 & 3,83 \\
\hline $\begin{array}{l}\text { pour effectuer des } \\
\text { opérations } \\
\text { bancaires }\end{array}$ & test & & & & 36) $=2,4$ & $1 ; \mathrm{p}<$ & 0,05 & & & & $F_{(4 ; 236)}=$ & 0,95 & $=0,4$ & \\
\hline J'utilise Internet & $\bar{X}$ & 2,17 & 1,61 & 2,0 & 1,52 & 1,84 & 1,55 & 3,00 & 1,43 & 1,73 & 1,62 & 2,27 & 1,79 & 1,67 \\
\hline $\begin{array}{l}\text { pour des fins de } \\
\text { téléphonie }\end{array}$ & test & & & & 37) $=2,9$ & $8 ; p<$ & 0,01 & & & & $\mathrm{~F}_{(4 ; 237)}=$ & 1,01 & $=0,4$ & \\
\hline J'utilise Internet & $\bar{X}$ & 2,89 & 2,50 & 3,8 & 3,33 & 3,34 & 2,41 & 3,65 & 2,38 & 2,93 & 3,02 & 3,64 & 3,16 & 3,00 \\
\hline $\begin{array}{l}\text { pour trouver des } \\
\text { informations sur la } \\
\text { santé }\end{array}$ & test & & & & 36) $=2,5$ & $2 ; p<$ & 0,05 & & & & $F_{(4 ; 236)}$ & 0,61 & $=0,6$ & \\
\hline J'utilise Internet & $\bar{X}$ & 2,12 & 1,91 & 2,7 & 1,95 & 1,67 & 1,45 & 2,06 & 1,93 & 1,67 & 1,76 & 2,97 & 1,72 & 2,83 \\
\hline $\begin{array}{l}\text { pour trouver des } \\
\text { informations sur la } \\
\text { bourse et les } \\
\text { finances }\end{array}$ & test & & & & 33) $=0,7$ & $2 ; p=$ & 0,49 & & & & $F_{(4 ; 233)}$ & 5,10 & $<0,0$ & \\
\hline J'utilise Internet & $\bar{X}$ & 2,39 & 3,14 & 3,1 & 3,03 & 3,42 & 2,79 & 3,24 & 2,41 & 2,71 & 2,80 & 3,56 & 3,18 & 4,00 \\
\hline $\begin{array}{l}\text { pour la } \\
\text { météorologie }\end{array}$ & test & & & & 36) $=0$, & $9 ; p=$ & 0,44 & & & & $\mathrm{~F}_{(4 ; 236)}=$ & 1,30 & $=0,2$ & \\
\hline & $\bar{X}$ & 3,65 & 3,76 & 4,0 & 4,25 & 4,66 & 3,59 & 4,81 & 3,32 & 3,97 & 3,65 & 4,35 & 4,54 & 4,00 \\
\hline $\begin{array}{l}\text { (e-mall) pour } \\
\text { communiquer avec } \\
\text { des ami-e-s }\end{array}$ & test & & & & 26) $=1,8$ & $2 ; p=$ & 0,08 & & & & $F_{(4 ; 226)}$ & 0,86 & $=0,4$ & \\
\hline J'utilise le courriel & $\bar{X}$ & 3,67 & 3,41 & 3,9 & 4,10 & 4,36 & 3,34 & 4,35 & 2,55 & 3,48 & 3,64 & 4,15 & 4,22 & 3,92 \\
\hline $\begin{array}{l}\text { (e-mail) pour } \\
\text { communiquer avec } \\
\text { les membres de ma } \\
\text { famille }\end{array}$ & test & & & & $37)=$ & 9 & & & & & $F_{(4 ; 237)}$ & 1,63 & $=0,1$ & \\
\hline J'envoie des & $\bar{X}$ & 3,00 & 3,86 & 2,7 & 3,33 & 4,68 & 2,62 & 2,67 & 2,17 & 2,38 & 3,17 & 3,33 & 4,49 & 4,64 \\
\hline $\begin{array}{l}\text { messages } \\
\text { électroniques dans } \\
\text { le cadre de mon } \\
\text { travail }\end{array}$ & test & & & & $28)=0$, & $3 ; \mathrm{p}$ &, 56 & & & & $\mathrm{~F}_{4 ; ; 28}$ & 4,61 & $<0,0$ & \\
\hline
\end{tabular}

Un troisième tableau attire l'attention sur l'attitude de l'individu à l'égard de l'informatique (il rapporte 3 des 9 énoncés sur lesquels ont porté les tests). Il ne signale pas d'inégalités de moyennes entre les professions, qu'il s'agisse du secteur ou du niveau. Cette fois, la valeur de «1 1 " correspond à " pas du tout d'accord " et celle de " 6 " à « tout à fait d'accord ». Compte tenu de la forme des énoncés, plus la valeur est faible, moins l'individu tend à être rebuté. Les moyennes sont communément inférieures à 4, mais supérieures à 3. Elles témoignent donc d'un certain malaise, mais non pas d'un handicap grave. 


\section{Tableau 3}

Valeur moyenne des attitudes à l'égard de l'informatique et d'Internet selon le secteur et le niveau de la profession

\begin{tabular}{|c|c|c|c|c|c|c|c|c|c|c|c|c|c|c|}
\hline \multirow[t]{2}{*}{ Attitude } & & \multicolumn{8}{|c|}{ Secteur de la profession } & \multicolumn{5}{|c|}{ Niveau de la profession } \\
\hline & & 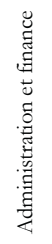 & 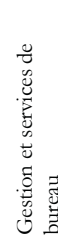 & 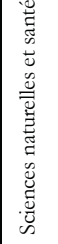 & 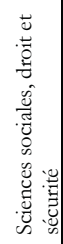 & 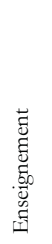 & 苞 & 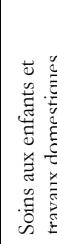 & 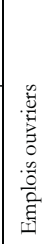 & 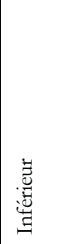 & 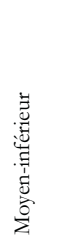 & $\frac{\mathrm{D}}{\mathrm{O}}$ & 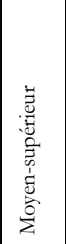 & 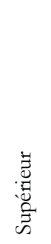 \\
\hline \multirow[b]{2}{*}{$\begin{array}{l}\text { Je solutionne } \\
\text { la plupart des } \\
\text { problèmes } \\
\text { techniques } \\
\text { que je } \\
\text { rencontre } \\
\text { quand } \\
\text { j'utilise un } \\
\text { ordinateur }\end{array}$} & $\bar{X}$ & 4,07 & 3,77 & 3,87 & 3,07 & 3,60 & 2,69 & 3,44 & 3,04 & 2,82 & 3,68 & 3,97 & 3,54 & 3,17 \\
\hline & test & & & \multicolumn{6}{|c|}{$\mathrm{F}_{(7 ; 218)}=1,53 ; \mathrm{p}=0,16$} & \multicolumn{5}{|c|}{$\mathrm{F}_{(4 ; 218)}=2,43 ; \mathrm{p}<0,05$} \\
\hline \multirow[b]{2}{*}{$\begin{array}{l}\text { J'ai le } \\
\text { sentiment, } \\
\text { quand je } \\
\text { manipule } \\
\text { mon } \\
\text { ordinateur, de } \\
\text { manquer de } \\
\text { connaissances } \\
\text { en } \\
\text { informatique }\end{array}$} & $\bar{X}$ & 3,50 & 2,74 & 3,04 & 3,51 & 3,51 & 3,84 & 4,35 & 3,40 & 3,83 & 3,25 & 2,95 & 3,42 & 3,92 \\
\hline & test & & & \multicolumn{6}{|c|}{$\mathrm{F}_{(7 ; 221)}=1,89 ; \mathrm{p}=0,07$} & \multicolumn{5}{|c|}{$\mathrm{F}_{(4 ; 221)}=0,55 ; \mathrm{p}=0,70$} \\
\hline \multirow{2}{*}{$\begin{array}{l}\text { Les } \\
\text { ordinateurs } \\
\text { me jouent des } \\
\text { tours } \\
\text { désagréables }\end{array}$} & $\bar{X}$ & 3,67 & 2,81 & 2,74 & 3,02 & 3,05 & 3,58 & 3,41 & 3,20 & 3,34 & 3,17 & 3,03 & 3,03 & 2,33 \\
\hline & test & \multicolumn{8}{|c|}{$\mathrm{F}_{(7 ; 214)}=0,81 ; \mathrm{p}=0,58$} & \multicolumn{5}{|c|}{$\mathrm{F}_{(4 ; 214)}=0,32 ; \mathrm{p}=0,87$} \\
\hline
\end{tabular}

Un quatrième tableau se concentre sur les objets d'identification sociale : la famille, le métier, le sexe, etc. (il présente 10 des 16 objets sur lesquels ont porté les tests). Une valeur de " 1 » indique une non-correspondance entre l'individu et l'objet, une valeur de " 6 », une forte identification à l'objet proposé. Le secteur professionnel est peu distinctif de l'opinion; le niveau l'est un peu plus. La famille et le pays interpellent davantage que l'organisme pour lequel on travaille. 


\begin{tabular}{|c|c|c|c|c|c|c|c|c|c|c|c|c|c|c|}
\hline & & & $\begin{array}{l}\mathrm{Va} \\
\text { elon } \\
1=\mathrm{p}\end{array}$ & eur & $\begin{array}{l}\text { oyen } \\
\text { eur et } \\
\text { ut d'ac }\end{array}$ & $\begin{array}{l}\text { able } \\
\text { he de } \\
\text { le ni } \\
\text { cord ; }\end{array}$ & $\begin{array}{l}\text { u } 4 \\
\text { obje } \\
\text { reau } \\
6=\text { to }\end{array}$ & $\begin{array}{l}\text { ts d'ic } \\
\text { e la } \\
\text { tt à fa }\end{array}$ & $\begin{array}{l}\text { lenti } \\
\text { rofe } \\
\text { t d'ac }\end{array}$ & $\begin{array}{l}\text { sion } \\
\text { ord) }\end{array}$ & & & & \\
\hline Objet & & & & Sec & ar de 1 & profe & ssion & & & & veau & e lap & ofessi & \\
\hline $\begin{array}{l}\text { Je } \\
\text { m'identifie... }\end{array}$ & & 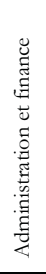 & 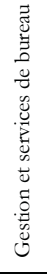 & 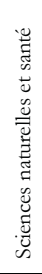 & 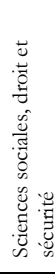 & 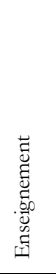 & 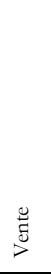 & 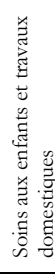 & 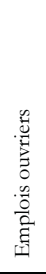 & 荀 & 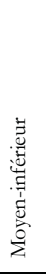 & 苛 & 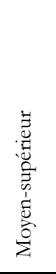 & 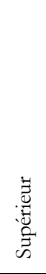 \\
\hline ... à ma famille & $\bar{X}$ & 5,59 & 5,71 & 5,25 & 5,77 & 5,68 & 5,74 & 5,65 & 5,38 & 5,65 & 5,50 & 5,87 & 5,71 & 5,00 \\
\hline & test & & & & ${ }_{4}=1,7$ & $3 ; p=$ & 0,10 & & & & $(4 ; 224)=$ & 2,95 & $p<0,0$ & \\
\hline$\ldots$ à la & $\bar{X}$ & 4,59 & 4,71 & 4,71 & 4,84 & 5,24 & 4,75 & 5,00 & 3,84 & 4,75 & 4,48 & 4,90 & 5,13 & 4,42 \\
\hline francophonie & test & & & & 1) $=1,0$ & $6 ; p=$ & 0,12 & & & & $(4 ; 220)=$ & 0,97 & $p=0,4$ & \\
\hline .... à l'Ontario & $\bar{X}$ & 4,53 & 4,10 & 4,00 & 4,56 & 4,62 & 4,62 & 4,88 & 4,00 & 4,53 & 4,41 & 4,23 & 4,65 & 3,50 \\
\hline français & test & & & & 3) $=2,0$ & $2 ; p=$ & 0,05 & & & & $(4 ; 223)=$ & 1,80 & $\mathrm{p}=0,1$ & \\
\hline & $\bar{X}$ & 4,67 & 4,10 & 4,00 & 4,32 & 4,11 & 4,28 & 4,76 & 3,76 & 4,28 & 4,36 & 4,42 & 4,08 & 3,33 \\
\hline $\begin{array}{l}\text { francophonie } \\
\text { du Nord-Ouest } \\
\text { de l'Ontario }\end{array}$ & test & & & & D) - & ; p & 0,05 & & & & $(4 ; 220)=$ & 2,01 & $=0,0$ & \\
\hline$\ldots$ au Nord- & $\bar{X}$ & 4,88 & 4,19 & 4,04 & 4,41 & 4,17 & 4,72 & 4,65 & 3,96 & 4,38 & 4,42 & 4,80 & 4,24 & 3,00 \\
\hline $\begin{array}{l}\text { Ouest de } \\
\text { l'Ontario }\end{array}$ & test & & & & 4) $=1, \mathrm{C}$ & $; p=$ & 0,13 & & & & $(4 ; 214)$ & 3,28 & $\mathrm{p}<0,0$ & \\
\hline .... à l'Ontario & $\bar{X}$ & 4,67 & 4,29 & 4,38 & 4,74 & 4,67 & 4,89 & 4,88 & 4,21 & 4,74 & 4,48 & 4,90 & 4,68 & 3,33 \\
\hline & test & & & & 4) $=1,7$ & $7 ; p=$ & 0,09 & & & & $(4 ; 214)$ & 2,52 & $\mathrm{p}<0,0$ & \\
\hline ... au Canada & $\bar{X}$ & 5,31 & 4,95 & 5,13 & 5,35 & 5,23 & 5,41 & 5,18 & 4,70 & 5,21 & 4,96 & 5,69 & 5,27 & 4,50 \\
\hline & test & & & & 4) $=0,8$ & $8 ; p=$ & 0,52 & & & & $(4 ; 214)$ & 2,16 & $p=0,0$ & \\
\hline ... au métier & $\bar{X}$ & 4,67 & 5,19 & 5,04 & 5,11 & 5,43 & 4,85 & 4,53 & 4,16 & 4,54 & 4,96 & 5,42 & 5,33 & 5,08 \\
\hline que j'exerce & test & & & & 7) $=0,9$ & $1 ; p=$ & 0,50 & & & & $(4 ; 217)=$ & 1,61 & $\mathrm{p}=0,1$ & \\
\hline & $\bar{X}$ & 4,00 & 5,19 & 4,68 & 4,84 & 5,05 & 4,96 & 4,19 & 3,81 & 4,37 & 4,94 & 5,21 & 4,73 & 4,92 \\
\hline $\begin{array}{l}\text { l'organisme } \\
\text { pour lequel je } \\
\text { travaille }\end{array}$ & test & & & & 1) $=1$ & $\cdot \mathrm{D}=$ & ,22 & & & & $(4 ; 211)=$ &, 51 & $<0,0$ & \\
\hline ... à mon sexe & $\bar{X}$ & 4,20 & 5,25 & 5,00 & 5,29 & 5,29 & 5,44 & 4,94 & 4,28 & 5,15 & 4,77 & 5,43 & 5,29 & 3,83 \\
\hline $\begin{array}{l}\text { (au fait d’être } \\
\text { homme ou } \\
\text { femme) }\end{array}$ & test & & & & $=2$ & $; p$ & & & & & $(4 ; 218)$ & 16 & $=0,0$ & \\
\hline
\end{tabular}

Un cinquième tableau se tourne vers l'engagement social. Une valeur de « 1 » témoigne d'une implication nulle, de « 6 », d'une dévotion marquée. Les tests (le tableau présente deux énoncés sur les six qui ont été testés) ne font pas état de variations significatives entre les professions et ils mettent en évidence la retenue des individus qui appartiennent à chacun des groupes comparés. 


\begin{tabular}{|c|c|c|c|c|c|c|c|c|c|c|c|c|c|c|}
\hline & & & $\begin{array}{l}\text { leur } \\
\text { elon } \\
1=p\end{array}$ & du t & $\begin{array}{l}\text { ne de } \\
\text { eur et } \\
\text { ut d'ac }\end{array}$ & $\begin{array}{l}\text { ablea } \\
\text { type } \\
\text { e niv } \\
\text { ord ; }\end{array}$ & $\begin{array}{l}5 \\
\text { d'im } \\
\text { eau de } \\
=\text { tou }\end{array}$ & $\begin{array}{l}\text { licati } \\
\text { la pr } \\
\text { à fait }\end{array}$ & $\begin{array}{l}\text { on sc } \\
\text { fess } \\
\text { l'acco }\end{array}$ & & & & & \\
\hline Type & & & & & eur de & prof & ssion & & & & Niveau & elap & ofessio & \\
\hline & & 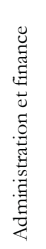 & 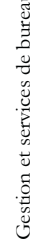 & 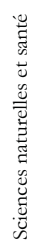 & 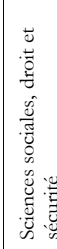 & 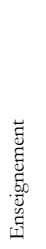 & 苞 & 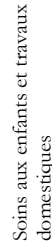 & 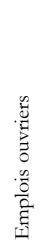 & 莺 & 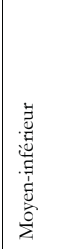 & 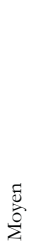 & 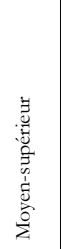 & 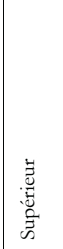 \\
\hline Je suis impliqué-e & $\bar{X}$ & 2,95 & 2,04 & 2,33 & 2,11 & 2,37 & 2,07 & 3,35 & 1,41 & 1,79 & 2,41 & 2,61 & 2,22 & 2,75 \\
\hline $\begin{array}{l}\text { dans des } \\
\text { organismes de } \\
\text { développement } \\
\text { communautaire } \\
\text { pour la } \\
\text { francophonie du } \\
\text { Nord-Ouest de } \\
\text { l'Ontario }\end{array}$ & test & & & & $2.35)=1$, & $4 ; p=$ & 0,10 & & & & $\mathrm{~F}_{(4,235)}$ & 1,72; & $=0,15$ & \\
\hline Je suis impliqué-e & $\bar{X}$ & 2,63 & 1,91 & 2,54 & 2,10 & 2,38 & 1,96 & 2,00 & 1,71 & 1,79 & 2,43 & 2,29 & 2,26 & 2,67 \\
\hline $\begin{array}{l}\text { dans des } \\
\text { organismes de } \\
\text { developpement } \\
\text { communautaire }\end{array}$ & test & & & & 234) $=1$, & $5 ; p=$ & & & & & $\mathrm{F}_{(4,234)}=$ & 1,90; & $=0,11$ & \\
\hline
\end{tabular}

Ces analyses démontrent qu'une théorie simpliste comme celle des classes sociales est peu en mesure de rendre compte des variations des usages des médias, de l'identité ou de l'engagement social, pour peu que le niveau de la profession constitue un indicateur de ces classes. On a affaire à des phénomènes qui réclament une interprétation beaucoup plus fine que celle que peut fournir cette théorie commune. Les analyses mettent aussi en évidence que les sociétés se constituent nettement sur le mode de la différenciation et de l'indifférenciation. Les égalités de moyennes rappellent le fait de la similitude, les inégalités, celui de la dissemblance (les écarts-types dont nous ne faisons pas ici mention auraient, eux aussi, signalé quelque différenciation).

Les principes de la systémique invitent à examiner les liens entre les divers objets qui ont été analysés, car il est peu probable qu'on ait affaire à un univers aux éléments tout à fait dissociés. D'autres analyses statistiques doivent alors être effectuées. Nous avons choisi de construire des matrices corrélationnelles (nous aurions pu procéder à des analyses de facteurs ou de regroupe- 
ments). Les résultats de ces corrélations sont synthétisés dans le tableau 6.

\section{Tableau 6}

Caractéristiques des divers ensembles d'élément intercorrélés

Éléments intercorrélés

- usages d'Internet

- usages des autres médias

- indicateurs d'identité

- indicateurs d'engagement

- Internet et autres médias

- Internet et identité

- autres médias et identité

- Internet et engagement

- autres médias et engagement

- engagement et identité
Caractéristiques des corrélations

- positives et assez fortes

- positives et assez faibles

- positives et assez fortes

- positives et assez fortes

- positives et assez faibles

- positives et faibles

- positives et faibles

- positives et faibles

- positives et faibles

- positives et assez faibles

Pour rendre compte de tous ces résultats, il faut les organiser en système. Nous proposons une logique comme celle que présente la figure 2. Chacun des composés que sont l'engagement social, l'identité et l'usage des médias est associé positivement aux deux autres et tous les trois forment un ensemble. Cet ensemble entretient une relation dialectique avec l'emploi en même temps que chacun de ses éléments composites est corrélé récursivement à l'emploi. Ce système, toutefois, est loin d'être suffisant, ce que soulignent les corrélations faibles; il est fortement déterminé par ce qui est autre que lui en même temps que cette altérité subit son influence. 


\section{Figure 2}

Ensemble conceptuel pour l'explication du rapport entre l'emploi, l'engagement social, l'identité et l'usage des médias

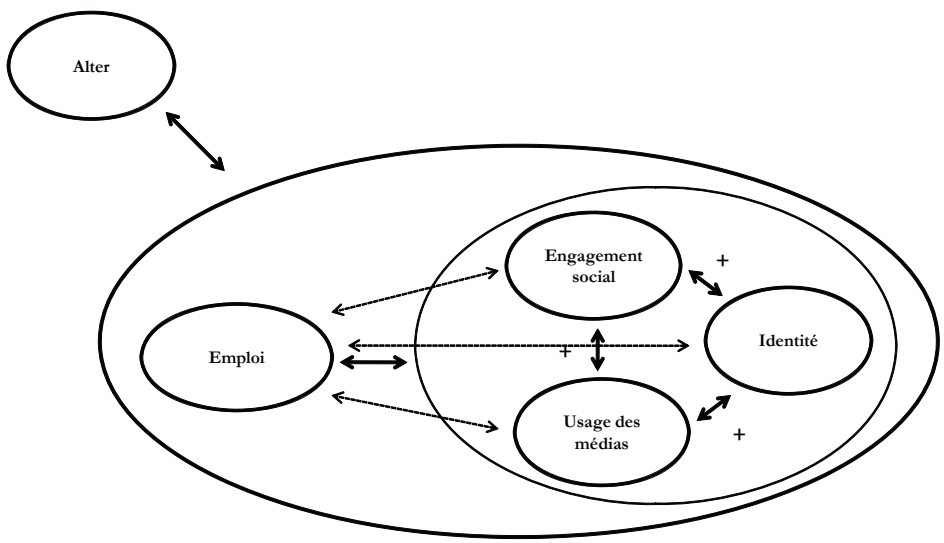

\subsection{Autour d'une analyse de régression}

Dans cette seconde analyse, il s'agit de découvrir les facteurs de l'affection pour la communauté où l'on résides. L'échantillon est formé de jeunes qui ont terminé leur $12^{\mathrm{e}}$ année deux ans avant la collecte de laquelle proviennent les données. Une analyse de régression par sélection identifie les variables pour lesquelles le lien est significatif. Ces variables sont des énoncés qui tantôt permettent de mesurer l'appréciation pour des objets ou pour des activités, tantôt donnent accès à la manière dont les jeunes se représentent le monde : les relations aux autres, la politique, la communauté... Des dizaines de variables sont ainsi testées. L'analyse (voir le tableau 7) en retient 11 qui expliquent $85 \%$

5 Ces analyses sont tirées d'une enquête longitudinale qui est menée dans le Nord-Est de l'Ontario. On les trouvera dans le rapport rédigé par Simon Laflamme et Pierre Bouchard intitulé Les jeunes et le nord: un parcours à découvrir $-3^{e}$ année (Hearst, Commission de formation du Nord-Est (CFNE) / Hearst, Collège Universitaire de Hearst / Sudbury, Université Laurentienne, http:/ / www.fnetbcfne.on.ca/French/Youth/LesjeunesetleNordabreg3.pdf, 2007. 
$\left(\mathrm{R}^{2}\right.$ total $\left.=0,85\right)$ de la variance des réponses associées à la proposition "j'aime ma communauté ". L'équation qui rassemble ces variables est inférable dans l'ensemble (ce dont témoigne la valeur de $\mathrm{F}$ et le $\mathrm{p}(<0,05)$ qui lui correspond). Elle révèle, par exemple, que plus le jeune estime que sa communauté offre suffisamment d'activités récréatives, plus il l'affectionne [puisque la valeur de $\beta(0,15)$ est positive], que plus il s'intéresse aux questions politiques de son école, plus il apprécie sa communauté [puisque la valeur de $\beta(0,32)$ est positive], que moins il aime les grandes villes, meilleurs sont ses sentiments pour sa communauté [puisque la valeur de $\beta(-0,48)$ est négative]. Et ainsi de suite.

\begin{tabular}{|c|c|c|c|}
\hline \multicolumn{4}{|c|}{$\begin{array}{c}\text { Tableau } 7 \\
\text { Régression multiple de diverses variables } \\
\text { dont l'influence est apparue significative } \\
\text { (appréciations et représentations) } \\
\text { pour l'énoncé « J'aime ma communauté » } \\
\text { Échantillon de } 2007 \text {, cohorte de } 12^{\mathrm{e}} \text { année seulement } \\
\left.\text { (Variance expliquée }\left(\mathrm{R}^{2}\right) \text { et coefficient standardisé }(\beta)\right) \\
\text { (Pour les énoncés relatifs à l'appréciation }: 1=\text { pas du tout et } 6=\text { beaucoup) } \\
\text { (Pour les énoncés relatifs aux représentations : } 1=\text { pas du tout d'accord et } 6=\text { tout à fait d'accord) }\end{array}$} \\
\hline Variable déterminante sélectionnée & $\beta$ & $\mathrm{F}$ & $\mathrm{p}<0,05$ \\
\hline Ma communauté offre suffisamment d'activités récréatives & 0,15 & 21,72 & oui \\
\hline Je m’intéresse aux questions politiques de mon école & 0,32 & & \\
\hline J'aime les grandes villes & $-0,48$ & & \\
\hline J'aime aller au cirque & 0,18 & & \\
\hline Je m’intéresse aux questions politiques du Canada & $-0,66$ & & \\
\hline Je m’intéresse aux questions politiques de ma communauté & 0,79 & & \\
\hline $\begin{array}{l}\text { J'ai beaucoup d'activités en dehors du cadre de mes études et } \\
\text { de mon travail }\end{array}$ & $-0,35$ & & \\
\hline Ma relation avec mes ami-e-s est harmonieuse & 0,20 & & \\
\hline J'aime assister à des événements sportifs & 0,32 & & \\
\hline Ma relation avec mes enseignant-e-s est harmonieuse & 0,21 & & \\
\hline $\begin{array}{l}\text { Il m'arrive de me sentir mal à l'aise quand je m'exprime en } \\
\text { anglais }\end{array}$ & 0,17 & & \\
\hline $\mathrm{R}^{2}$ total $=0,85$ & & & \\
\hline
\end{tabular}

Cette série d'onze corrélations appelle une interprétation. L'évocation de l'équation de régression dans sa forme stricte $\mathrm{Y}=\mathrm{b}_{0}+\mathrm{b}_{1} \mathrm{X}_{1}+\mathrm{b}_{2} \mathrm{X}_{2}-\mathrm{b}_{3} \mathrm{X}_{3}+\mathrm{b}_{4} \mathrm{X}_{4}-\mathrm{b}_{5} \mathrm{X}_{5}+\mathrm{b}_{6} \mathrm{X}_{6}-\mathrm{b}_{7} \mathrm{X}_{7}+\mathrm{b}_{8} \mathrm{X}_{8}$ $+\mathrm{b}_{9} \mathrm{X}_{9}+\mathrm{b}_{10} \mathrm{X}_{10}+\mathrm{b}_{11} \mathrm{X}_{11}+$ ALTER

ne permet pas d'appréhender toute l'information qui est manipulée. 
Elle donne l'illusion d'une logique strictement additive ou de rapports séparés et unidirectionnels entre la variable dépendante et chacune des variables indépendantes, un peu comme les représente le schéma suivant :

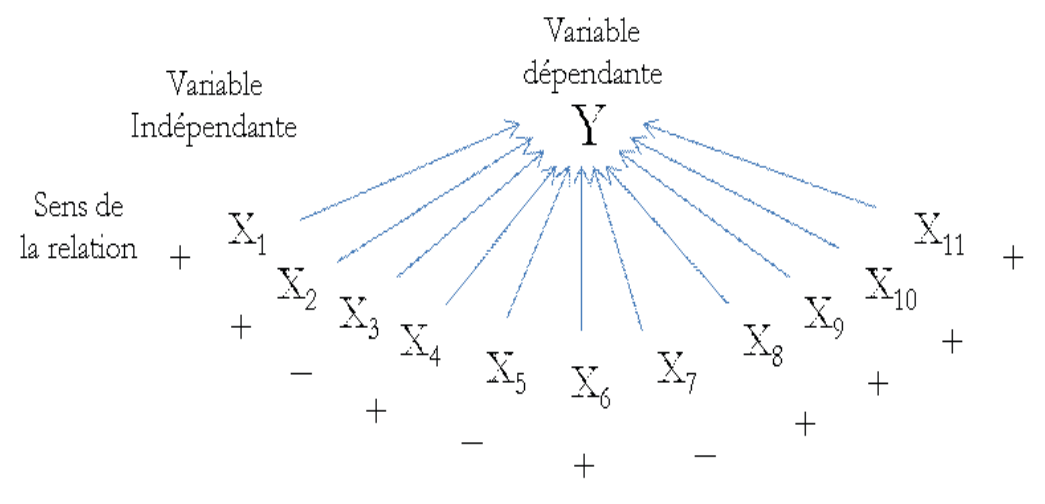

alors que l'analyse a pris en considération le poids relatif de toutes les variables les unes par rapport aux autres, c'est-à-dire qu'elle a calculé les dynamiques de toutes les variables indépendantes les unes par apport aux autres dans leur relation avec la variable dépendante. En fait, l'analyse de régression a intégré les variances expliquées dans un ensemble intercorréllé de variables indépendantes, et c'est grâce à cette logique, qui se comprend mieux avec les diagrammes de Venn qu'avec le schéma précédent, qu'elle parvient à exclure des variables et à en retenir d'autres. Rappelons au passage que la variance expliquée n'est qu'une corrélation au carré.

En prenant en considération les résultats que livre l'analyse de régression multiple (modifications dans la variance expliquée au fur et à mesure de l'ajout de variables dans l'équation, corrélations partielles ou corrélations semi-partielles, tolérances des variables indépendantes), on peut déjà obtenir plusieurs renseignements sur les rapports qu'entretiennent les variables entre elles; mais on peut aussi tout simplement insérer les variables indépendantes dans une matrice intercorrélationnelle. On note alors (voir le tableau 8) que les relations sont parfois assez fortes (supérieures 
à 0,50 ) et qu'elles sont souvent très faibles.

\begin{tabular}{|c|c|c|c|c|c|c|c|c|c|c|}
\hline \multicolumn{11}{|c|}{$\begin{aligned} \text { Tableau } 8 \\
\text { Matrice des corrélations entre les variables associ }\end{aligned}$} \\
\hline & 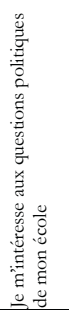 & 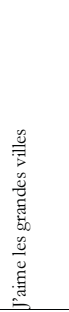 & 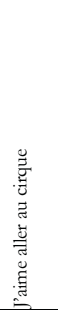 & 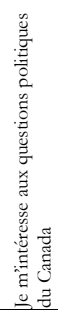 & 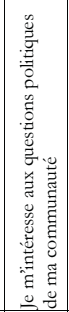 & 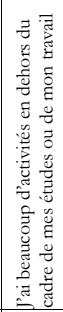 & 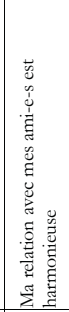 & 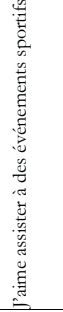 & 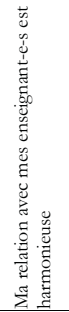 & 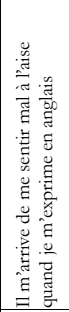 \\
\hline $\begin{array}{l}\text { Ma communauté offre } \\
\text { suffisamment d'activités } \\
\text { récréatives }\end{array}$ & 0,22 & $-0,04$ & 0,25 & 0,19 & 0,19 & 0,27 & 0,07 & 0,10 & 0,16 & 0,10 \\
\hline $\begin{array}{l}\text { Je m'intéresse aux questions } \\
\text { politiques de mon école }\end{array}$ & & 0,11 & 0,08 & 0,55 & 0,59 & 0,20 & $-0,04$ & $-0,02$ & 0,21 & 0,04 \\
\hline J'aime les grandes villes & & & 0,14 & 0,09 & \begin{tabular}{|l|}
0,03 \\
\end{tabular} & \begin{tabular}{|l|}
0,11 \\
\end{tabular} & 0,14 & 0,14 & 0,13 & $-0,04$ \\
\hline J'aime aller au cirque & & & & 0,09 & 0,10 & 0,16 & 0,02 & 0,16 & 0,04 & 0,09 \\
\hline $\begin{array}{l}\text { Je m'intéresse aux questions } \\
\text { politiques du Canada }\end{array}$ & & & & & 0,68 & 0,15 & $-0,01$ & $-0,08$ & 0,18 & 0,03 \\
\hline $\begin{array}{l}\text { Je m'intéresse aux questions } \\
\text { politiques de ma communauté }\end{array}$ & & & & & & 0,18 & $-0,07$ & 0,02 & 0,13 & 0,06 \\
\hline $\begin{array}{l}\text { J'ai beaucoup d'activités en } \\
\text { dehors du cadre de mes études } \\
\text { et de mon travail }\end{array}$ & & & & & & & $0,17 *$ & 0,32 & 0,20 & $-0,05$ \\
\hline $\begin{array}{l}\text { Ma relation avec mes ami-e-s est } \\
\text { harmonieuse }\end{array}$ & & & & & & & & 0,20 & 0,32 & $-0,07$ \\
\hline $\begin{array}{l}\text { J'aime assister à des événements } \\
\text { sportifs }\end{array}$ & & & & & & & & & $-0,05$ & $-0,03$ \\
\hline $\begin{array}{l}\text { Ma relation avec mes } \\
\text { enseignant-e-s est harmonieuse }\end{array}$ & & & & & & & & & & $<0,01$ \\
\hline
\end{tabular}

En tenant compte de ces valeurs, on peut regrouper les variables en invoquant des raisons sémantiques ou théoriques. (Une analyse de facteurs aurait pu fournir des motifs statistiques pour procéder à des interprétations sémantiques.) Trois ensembles peuvent être définis : l'un qui a trait à l'engagement social, un deuxième à ce que la communauté a à offrir et un troisième aux relations avec les autres. À ces trois ensembles, compte tenu de l'incomplétude de la variance expliquée, il semble qu'on doive ajouter un registre de l'altérité, c'est-à-dire de ce qui n'est pas pris en charge par l'analyse. On peut alors dessiner le système suivant (organisation de l'information qui n'est pas exclusive) : 
Figure 3

Ensemble conceptuel pour

l'explication de l'affection pour la communauté

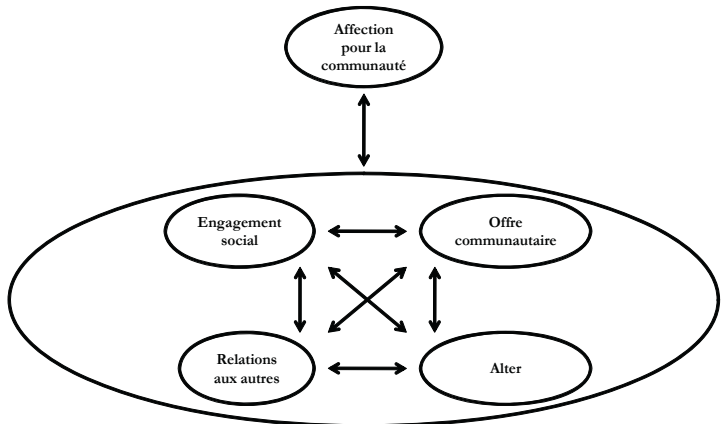

La figure montre que l'affection pour la communauté dépend de l'interrelation de l'engagement social, des relations aux autres et de la perception de ce que la communauté a à offrir. Plus le jeune s'engage socialement, alors meilleures sont ses relations aux autres et plus il est satisfait de ce que la communauté lui offre; meilleures sont ses relations aux autres, alors plus il s'engage socialement et meilleure est sa perception de ce que lui offre sa communauté; plus il estime ce que sa communauté lui offre, alors plus il s'engage socialement et meilleures sont ses relations aux autres. De cette dynamique dépend l'affection pour la communauté. Ce triple ensemble n'est pas exclusif, ce pourquoi il faut rappeler l'apport de l'altérité. En fait, cette interprétation rappelle que plus le jeune travaille à modeler son environnement social, plus il est satisfait de son milieu et de ses relations aux autres et qu'on est ici dans une logique dialectique.

\section{Conclusion}

Qu'elle donne lieu à des comparaisons de moyennes ou à des régressions, la statistique offre aux chercheurs des informations auxquelles il faut donner une interprétation. En outre, la statis- 
tique, par la puissance de ses outils, est en mesure de gérer de nombreuses variables. Dès lors que ces variables donnent à observer un certain nombre des aspects de la socialité et que ces aspects sont corrélés, il faut en organiser logiquement les relations. La systémique vient alors au service du théoricien qui a su employer les outils statistiques. À l'inverse, le chercheur qui connaît les principes de la systémique peut employer la statistique pour construire l'ensemble organisé des aspects de la réalité sur lesquels il se penche.

En elle-même, la statistique est pauvre puisqu'elle n'est qu'une manière d'observer le monde que dessinent les modélisations. Elle est aussi pauvre que toute analyse à laquelle fait défaut l'interprétation, fût elle qualitative.

La systémique n'est attachée de façon immédiate à aucun type d'analyse en sciences sociales. La statistique, si limitée soit-elle, ne lui est pas plus interdite que les autres formes d'accès à l'empirie.

\section{Bibliographie}

Gauthier, Yvon, Entre science et culture. Introduction à la philosophie des sciences, Montréal, Presses de l'Université de Montréal, 2005.

Laflamme, Simon et Chris Southcott, "Le rapport aux médias et la profession exercée. Le cas des francophones du Nord-Ouest de l'Ontario ", à paraître.

Laflamme, Simon et Pierre Bouchard, Les jeunes et le nord: un parcours à découvrir - $3^{e}$ année (Hearst, Commission de formation du Nord-Est (CFNE) / Hearst, Collège Universitaire de Hearst / Sudbury, Université Laurentienne, http://www.fnetbcfne.on.ca/French/Youth/ LesjeunesetleNordabreg3.pdf, 2007, site consulté le 6 août 2008.

Morin, Edgar et Jean-Louis Le Moigne, L’intelligence de la complexité, Paris, L'Harmattan, coll. « Cognition et formation », 1999. 